\title{
Acute Total Occlusion of the Left Circumflex Coronary Artery Presenting with Non-ST-segment Elevation Myocardial Infraction and Normal Electrocardiogram - A Case Report
}

\author{
Teddy Arnold Sihite*(D), Sindy Hendrawansyah (D), Raymond Pranata (i) \\ Department of Cardiology and Vascular Medicine, Faculty of Medicine, Universitas Padjadjaran, Rumah Sakit Umum Pusat \\ Hasan Sadikin, Bandung, Indonesia
}

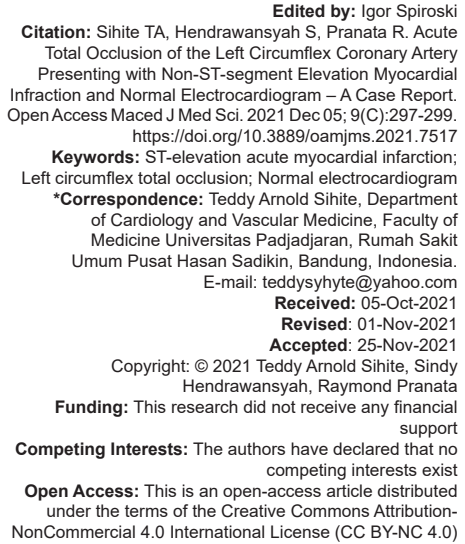

\begin{abstract}
BACKGROUND: In this case report, we report a patient with non-ST-segment elevation myocardial infarction (NSTEMI), presenting with recurrent chest pain typical of angina, a very high troponin I level despite normal electrocardiogram (ECG). On angiography, it turns out that the patient has acute total occlusion in the left circumflex artery (LCX).

CASE REPORT: A 56-year-old woman presented to the emergency department with chief complaint of recurrent chest pain typical of angina $20 \mathrm{~h}$ before admission. Vital signs were within normal limit. There were no murmur, additional heart sounds, and no rales or crackles. The ECG showed normal sinus rhythm, and there were no ST-T changes on serial examination. The first and second cardiac enzymes troponin I was high (>10 mg/L). Chest X-ray examination showed cardiomegaly without signs of lung edema. Patient was diagnosed with high-risk NSTEMI, hypertensive heart disease, and diabetes mellitus. Coronary showed an acute total occlusion in the LCx, which is determined as the culprit lesion for the ongoing myocardial infarction. A drug-eluting stent was deployed at the culprit lesion and the coronary flow was TIMI flow 3 . There was non-significant stenosis at the mid-right coronary artery. The echocardiography showed reduced left ventricular systolic function (LVEF 50\%) with hypokinetic inferior-septal and inferior-lateral segment base to apical. Post-procedural follow-up was uneventful.

CONCLUSION: One of the learning points is that ECG may fail to detect acute total occlusion and rise in troponin level, despite the absence of ST-T changes, warrant urgent invasive strategy.
\end{abstract}

Introduction

Acute total occlusion of coronary artery causing myocardial infarction manifest as ST-segment elevation acute myocardial infarction (STEMI) and reperfusion is the cornerstone of STEMI management [1]. Timely reperfusion or revascularization of the acute total occlusion is critical for myocardial survival. The efficacy of reperfusion therapy may decline over time because of decreased viable myocardium [2]. Thus, prompt diagnosis and treatment are of utmost importance.

Questions arose when patients with acute total occlusion of the coronary artery presented without signs of ST-segment elevation. This may delay treatment, especially in the area with limited resources. In ideal settings, patients presenting with non-ST-segment elevation myocardial infarction (NSTEMI) will undergo coronary angiography; although the procedure might be slightly delayed, it may be performed within a reasonable timeframe. Several ECG signs, such as De Winter ECG, may indicate the presence of total occlusion despite the absence of ST-segment elevation [3].
In this case report, we report a patient with NSTEMI, with recurrent chest pain typical of angina, a very high troponin I level despite a normal ECG. On angiography, it turns out that the patient has acute total occlusion in the left circumflex artery (LCX).

\section{Case Presentation}

A56-year-old woman presented to the emergency department with a chief complaint of recurrent chest pain since $20 \mathrm{~h}$ before admission. The pain radiated to back and jaw, and accompanied with heavy sweating. There was no history of dyspnea on effort, paroxysmal nocturnal dyspnea, orthopnea, or ankle edema. The patient had history of uncontrolled hypertension since 4 years before admission and diabetes. There was no history of smoking. The patient was no longer menstruating.

In the emergency department, she was moderately ill, fully alert, and had no dyspnea with 
moderate work of breathing. The blood pressure was $130 / 80 \mathrm{mmHg}$, heart rate $96 \mathrm{x} / \mathrm{m}$ of resting heart rate, regularly and equal, respiratory rate of $20 \mathrm{x} /$ minute, and with normal temperature, oxygen saturation was $99 \%$ on room air. There were no signs of heart failure on physical examination. There were no murmur, additional heart sounds, and no rales or crackles.

The electrocardiography (ECG) showed normal sinus rhythm, normal axis, and no ST-segment elevation in any lead, including RV lead and posterior, there were no ST-T changes on serial examination (Figure 1). Routine blood examination, ureum, creatinine, glucose, and electrolyte panel were within normal limits. The first and second cardiac enzymes troponin I was high (>10 mg/L). Chest X-ray examination showed cardiomegaly without signs of lung edema (Figure 2).

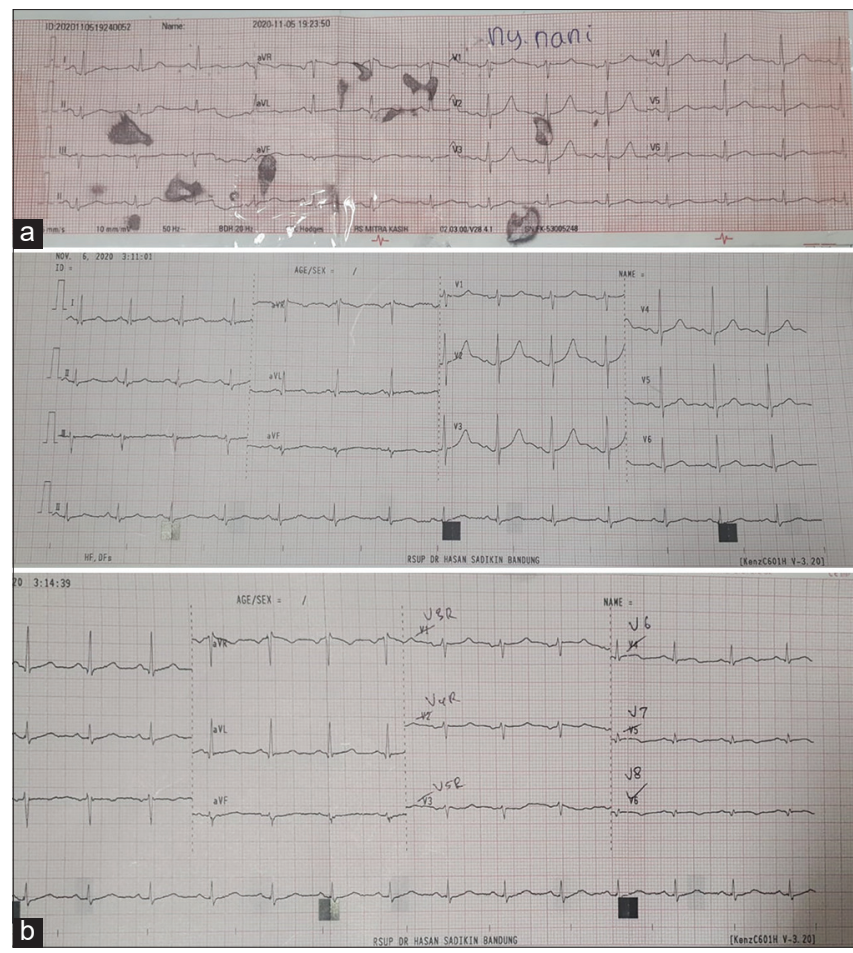

Figure 1: (a) ECG 15 hours from onset: Normal sinus rhythm. (b) ECG $20 \mathrm{~h}$ from onset: Normal sinus rhythm, no ST segment elevation, and nor ST-T dynamic changes

The patient was diagnosed with high-risk NSTEMI, hypertensive heart disease, and diabetes mellitus. Coronary showed an acute total occlusion in the LCx, which is determined as the culprit lesion for

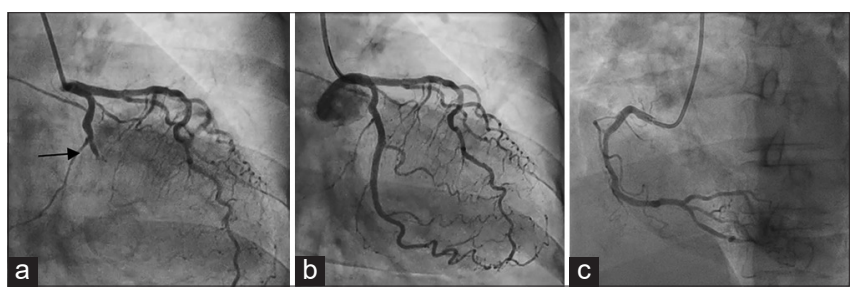

Figure 2: Coronary angiogram showed: (a) Acute total occlusion at proximal LCX (arrow); (b) Post DES insertion showed a good flow in the LCx; and (c) Non-significant stenosis at mid-right coronary artery the ongoing myocardial infarction (Figure 2a). A drugeluting stent (DES) was deployed at the culprit lesion, and the coronary flow was restored completely (TIMI Flow 3) (Figure 2b). There was non-significant stenosis at mid-right coronary artery (Figure 2c). After the procedure, the patient was transferred to the intensive cardiac care unit (ICCU).

The echocardiography showed normal all chambers with concentric left ventricle hypertrophy, reduced left ventricle systolic function (LVEF $50 \%$ biplane Simpson's) with hypokinetic inferior-septal and inferior-lateral segment base to apical, grade I Diastolic dysfunction, normal valves, low probability of $\mathrm{PH}$, normal RV contractility. Post-procedural follow-up was uneventful, the patient was hemodynamically stable, and was discharged with good condition.

\section{Discussion}

This case report showed NSTEMI patient with chest pain typical of angina and a very high troponin I level despite normal ECG. On angiography, it turns out that the patient has acute total occlusion in the LCx. Thus, ECG may fail to detect acute total occlusion and rise-and-fall in troponin level, despite the absence of ST-T changes in normal and additional leads, warrant urgent invasive strategy.

There are numerous possible causes of unequivocal ECG in LCx-related AMI. The first possible reason is due to the small infarct size. A previous study showed that a total mass of myocardium lost in LCx-related AMI is smaller than in other anatomic distributions, notably anterior MI. Infarct size could be estimated by the amount of serum cardiac marker released and decreased regional wall motion contractility [4]. However, our patient had significantly increased cardiac marker troponin I > $10 \mathrm{mg} / \mathrm{dl}$. It was possible that the infarct size was large, as evidenced by the elevated cardiac markers; however, it is ambiguous due to normal ECG findings. Several studies showed that patients without ST-segment changes were likely due to incomplete coronary occlusion due to thrombus or vasospasm [4]. However, coronary angiography suggests the absence of vasospasm or incomplete coronary occlusion. The coronary artery dominance may also obscure the ECG finding in LCx-related AMI. Right coronary dominance may act as a protective factor in acute occlusion of LCx by giving collateral or dual flow and minimize infarcted area which causes minimal changes in ECG recording [4]. Coronary angiography showed right dominance in our patient, the lateral posterior segment of myocardium in our patient might be protected by the right dominant coronary 
artery system. Another possible cause is the position of LCx, if LCx lies in the true posterior left ventricle region, it may cause difficulty in detecting ischemia due to air in posterior mediastinum, which is a poor conductor of electricity [5]. Finally, the normal ECG might be because transient ST-elevation myocardial infarction in ECG which was not recorded before admission.

Data from several reports showed that LCx is the least frequent culprit artery [4], [5], [6]. Failure to detect $L C x$ related $A M I$ may have great consequences because LCx supplies significant left ventricle areas [6]. An LCx supplies the inferior-basal area of the myocardium. In the ECG, the posterior leads, reflecting the basal part of the left ventricle wall, which lies on the diaphragm, which can be easily detected by posterior leads V7-V9 in the back. Because the anterior ECG leads are relatively in the opposite direction of the inferior-basal leads, an anterior ST depression is often the mirror image of an inferior-basal ST elevation. None of the 12 standard ECG leads reflect the inferior-basal wall, therefore, an isolated inferior-basal STEMI often masquerades as an NSTEMI [7]. The ESC STEMI 2017 guideline suggests that when we encounter a patient with ACS and normal 12 lead ECG, it is recommended to perform extra posterior leads $\mathrm{V} 7-\mathrm{V} 9$ recording to detect ST-elevation or ST-T changes in posterior leads. In our case, we have performed right and posterior ECG leads suggestive of normal ECG.

If in doubt regarding the possibility of acute evolving $\mathrm{MI}$ such as normal standard ECG and no recurrent chest pain found, emergency imaging aids the provision of timely reperfusion therapy to these patients. The European guideline recommends using echocardiography for initial diagnosis to find new regional wall motion abnormalities (RWMA). They concluded that emergency echocardiography before coronary angiography should be considered if the diagnosis is uncertain [1]. However, this modality is also imperfect because echocardiography could not differentiate a new RWMA from an old one. If echocardiography is not available or if doubts persist after echo, an urgent invasive strategy is warranted [1].

Left circumflex artery-related acute myocardial infarction has been known to be underdiagnosed with 12-lead ECG. Kim et al. conducted a study that focuses on the prognosis of occlusion in the three coronary vessels. There was no significant statistical difference among the three vessels in terms of in-hospital mortality. Multivariate analysis showed primary $\mathrm{PCl}$ decreased hospital mortality in patients with occluded coronary artery. In conclusion, LCx occlusion with normal ECG must be treated similarly to occlusion of other coronary arteries.

\section{Conclusion}

In this case report, we report a patient with NSTEMI, having chest pain typical of angina, a very high troponin I level despite normal ECG. One of the main reasons for not ST-segment elevation is that $L C x$ lies in the true posterior left ventricle region, causing difficulty in detecting ischemia due to air in posterior mediastinum, a poor conductor of electricity. On angiography, it turns out that the patient has acute total occlusion in the LCx. One of the learning points is that ECG may fail to detect acute total occlusion and rise in troponin level, despite the absence of ST-T changes, warrant an urgent invasive strategy.

\section{References}

1. Ibanez B, James S, Agewall S, Antunes MJ, Bucciarelli-Ducci C Bueno H, et al. 2017 ESC Guidelines for the management of acute myocardial infarction in patients presenting with ST-segment elevation: The task force for the management of acute myocardial infarction in patients presenting with ST-segment elevation of the European society of cardiology (ESC). Eur Heart J. 2018;39(2):119-77. https://doi.org/10.1093/eurheartj/ehx393 PMid:28886621

2. Antman EM. Time is muscle: Translation into practice. $\mathrm{J}$ Am Coll Cardiol. 2008;52(15):1216-21. https://doi.org/10.1016/j. jacc.2008.07.011

PMid:18926324

3. Pranata R, Huang I, Damay V. Should de winter T-wave electrocardiography pattern be treated as ST-segment elevation myocardial infarction equivalent with consequent reperfusion? A Dilemmatic experience in rural area of Indonesia. Case Rep Cardiol. 2018;2018:6868204. https://doi.org/10.1155/2018/6868204 PMid:29850267

4. Kim SS, Choi HS, Jeong MH, Cho JG, Ahn YK, Kim JH, et al Clinical outcomes of acute myocardial infarction with occluded left circumflex artery. J Cardiol. 2011;57(3):290-6. https://doi. org/10.1016/j.jjcc.2011.01.014 PMid:21388789

5. Turnipseed SD, Trythall WS, Diercks DB, Laurin EG, Kirk JD, Smith DS, et al. Frequency of acute coronary syndrome in patients with normal electrocardiogram performed during presence or absence of chest pain. Acad Emerg Med. 2009;16(6):495-9. https://doi.org/10.1111/j.1553-2712.2009.00420.x

PMid:19426294

6. FromAM, BestPJ, Lennon RJ, RihalCS, PrasadA.Acutemyocardia infarction due to left circumflex artery occlusion and significance of st-segment elevation. Am J Cardiol. 2010;106(8):1081-5. https:// doi.org/10.1016/j.amjcard.2010.06.016 PMid:20920642

7. Wong CK, White HD. Patients with circumflex occlusions miss out on reperfusion: How to recognize and manage them. Curr Opin Cardiol. 2012;27(4):327-30. https://doi.org/10.1097/ HCO.0b013e32835482b7

PMid:22565144 